\title{
The Physico-Chemical Water Quality of the Obafemi Awolowo University Teaching and Research Farm Lake, Ile-Ife, Southwest, Nigeria
}

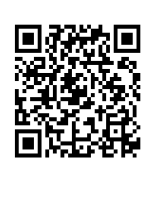

\author{
Aduwo A I* and Adeniyi I F \\ Limnology and Hydrobiology Laboratory, Zoology Department, Obafemi Awolowo University, Nigeria
}

Submission: April 02, 2019; Published: April 22, 2019

Corresponding author: Aduwo A I, Limnology and Hydrobiology Laboratory, Zoology Department, Obafemi Awolowo University, Ile-Ife, Osun State, Nigeria

\begin{abstract}
The physico-chemical water quality of the Obafemi Awolowo University Teaching and Research Farm Lake, Ile-Ife, Nigeria, was studied over an annual cycle (September 2006 to August 2007) almost 40 years after its impoundment in 1967. This was with a view to obtaining the then current state of physico-chemical water quality parameters with their variations in time and space as well as using some of the data obtained to evaluate the aging process of the lake for the 40 years of its existence. There was no significant ( $p>0.05$ ) horizontal variation (i.e. upstream dowstream) in the physico-chemical characteristics of the lake whereas, Apparent-colour, True colour, Turbidity, Total Suspended Solids (TSS), Total Solids (TS) and Total Acidity increased significantly $(\mathrm{p}<0.05)$ from the lake surface towards the bottom of the lake. In the other hand, water pH, Dissolved Oxygen (DO); Dissolved Oxygen Saturation (DO\% Sat.) and Biochemical Oxygen Demand (BOD5) decreased significantly ( $<<0.05$ ) from the lake surface towards the bottom of the lake.

Dissolved Oxygen (DO), Dissolved Oxygen Saturation (DO\% Sat.) and Nitrate ion (NO3-) were significantly higher ( $<<0.05)$ in the dry season than in the rainy season while, True colour, $\mathrm{pH}$, Conductivity, Total Hardness, Calcium ion (Ca2+), Magnesium ion (Mg2+), Potassium ion $(\mathrm{K}+)$ and Sulphate ion $(\mathrm{SO} 42-)$ were significantly higher $(\mathrm{p}<0.05)$ in the rainy season than in the dry season. The lake water could be regarded shallow, colored, turbid, less transparent, slightly acidic, soft and well-buffered fresh-waterbodies with evidence of aging (i.e. transforming the lake from an oligotrophic status to a eutrophic waterbody). The results indicated that the lake is suitable for most of its applications with little or no adjustments.
\end{abstract}

Keywords: Inundation; Oligotrophic; Eutrophic; Aging; Man-made; SpillwayUrbanization

\section{Introduction}

Water is probably the most important natural resource in the world, since without its life cannot exist and industries cannot operate. Unlike many other resources and/or raw materials, there is no substitute for water in many of its uses. Water plays a vital role in the development of communities since a reliable supply of water is an essential prerequisite for the establishment of a permanent community [1]. Water intended for human consumption should be "safe" and "wholesome" i.e. free from pathogenic agent and harmful chemicals, pleasant to taste and usable for domestic purpose [2]. The immense importance of water in our daily life makes it imperative that thorough examinations be conducted on it for most of its uses. The examinations mostly conducted are under the broad headings viz: physical, chemical and biological analysis. Physical analysis involves measurements of temperature, colour, odour and taste, solids and turbidity. Chemical analyses involve the measurements of cations, anions, nutrient compounds, toxic and non-toxic compounds and, of course tests for oxygen demands by inorganic and organic substances [3].
Boyd (1981) defined water quality as any characteristics of water, whether physical, chemical and biological, that can also affect the survival, reproduction, growth and management of fish. However more stringent control of water contaminants and higher quality standards apply to water intended for human consumption than for other uses. Compounds such as phosphate and nitrate are two important nutrients in the lake loading through point and non-point pollution sources such as washing, bathing, agricultural activities in fringe area, joining of domestic raw sewage, leading to the cultivation of water caltrop (Trapa spp.) and huge growth of aquatic macrophytes. These nutrients have been found to support the fast growth of aquatic plants (such as: water hyacinth (Eichhornia crassipes), Esthwaite waterweed (Hydrilla spp.), hornwort (Ceratophyllum spp. etc.) as a result the abundance of these plants lead to gradual shrinking of the lake area along with other complications like low light penetration, reduces oxygen concentration, clogging of water channels, lowers entertainment value of lake and sometimes the depletion of the oxygen level can lead to fish mortality also [2]. 
The Obafemi Awolowo University Teaching and Research Farm Lake was impounded between March and July 1967 majorly for agricultural purposes like irrigation and fish farming by regular stocking of the lake with different fish species. Adeniyi [4] indicated that fish in the lake survive on the natural food materials supplied through the two inlets (tributaries) and that the lake is often colored darkish brown due to the abundance of plankton life. Besides, in 1969 the following compounds were recommended by the Fisheries Division, Ministry of Agriculture and Natural Resources, Ibadan as manure for the lake

a. About 4 standard head-pans of poultry droppings per acre per month. This was spread uniformly over the lake for some periods in 1969 and 1970.

b. Four pound of Triple superphosphate per acre per month for a period of six months. This was also done for a while and later cancelled due to experiment being conducted by the Zoology Department of the then University of Ife.

The lake was also drained several times in 1970 due to cropping activities and this has continued till present. However, Aderounmu \& Adeniyi [5] observed that in 1970, the average minimum depth of $1.37 \mathrm{~m}$ was recorded for the lake in the month of April 1970, before drainage began; and the average maximum depth of $5.70 \mathrm{~m}$ was recorded in September and October 1970. This present study was conducted between September, 2006 and August, 2007 almost 40 years after its impoundment in 1967 with a view to obtaining the then current state of physico-chemical water quality parameters with their variations in time and space as well as using some of the data obtained to evaluate the aging process of the lake for the 40 years of its existence.

\section{Materials and Methods}

\section{Area of Study}

Ile-Ife is an ancient town in Southwestern Nigeria in which Obafemi Awolowo University formerly known as University of Ife is situated. The Faculty of Agriculture of the University had set apart the Teaching and Research Farm for the purpose of teaching students on the practical aspects of their courses as well as research at various capacities for education, growth and development. The studied lake is then situated in the Farm within the University. Ile-Ife and its immediate satellite villages lie at approximately Latitudes $07^{0} 26^{\prime}-07^{0} 33^{\prime} \mathrm{N}$ of the Equator and Longitudes $004^{0} 30^{/}-004^{0} 35^{/} \mathrm{E}$ of the Prime Meridian at mean altitude of roughly $300 \pm 50 \mathrm{~m}$ above the mean sea level. Ife is about $40 \mathrm{~km}$ south of Osogbo, the Osun State Capital, about 120 $\mathrm{km}$ north of the Atlantic Coast, and about $600 \mathrm{~km}$ southwest of Abuja the Federal Capital of Nigeria [6]

The lake is man-made being formed by the inundation of the area above the dam constructed a little below the confluence of two streams Elerin which flows between two villages, Kajola and Obagbile villages and its tributary, Omifunfun stream, and both flow from a relatively higher plains through a secondary forest. The lake is situated north to north-east of the University Campus with a wavy-line trapezium basin shape, occupies an approximate area of 11 acres at maximum fill [4]. The dam wall lies at the valley below the Poultry unit of the farm just immediately after The Faculty of Pharmacy drug research garden. The dammed end is being provided with both auxillary and mechanical spillways for the drainage and regulation of excess water. Fluctuation of water levels is always gradual as the shoreline slopes gently except at the dam area where the slope is steep.

The climate of the Ile-Ife area is of the Moist Monsoon Equatorial type $[7,8]$. Two major seasons prevail in the area, which are: the dry season and the rainy season. The dry season extends from November to March while the rainy season covers the remaining part of the year [9]. The average rainfall is about 1433 $\pm 256 \mathrm{~mm}$ (1955 - 1998) with a rainfall surplus of less than 1000 $\mathrm{mm}$. The annual regime is characterized by two peaks (occurring mostly in July and September) separated by a relatively dry spell in August commonly referred to as 'August break' [10]. Ambient air temperatures are moderately high throughout the year with maximum temperatures $\left(32.2-34.4^{\circ} \mathrm{C}\right)$ usually occurring in JulySeptember [6].

The area lies within the rainforest zone of Nigeria. It is mainly a lowland rainforest, with some areas of derived grassland [11]. The forest subtype is the dry deciduous forest [12]. The vegetation is that of the Guinean Congolean forest [13]. The lake area itself has a narrow shoreline even during dry season and the marginal vegetation consist of grass and water course community. Dominant among the marginal plant species are: Aneilema beniniense which tend to encroach on the water; and Axonopus compresus which was intentionally planted and maintained by regular cutting. The marginal vegetation gradually leads to a thin stretch of regrowth community which is bordered by the vegetable garden and plant nursery along the path leading from the Poultry unit and either cocoa plantation or secondary forests [4]. Ile-Ife area is underlain by the Pre-Cambrian Basement Complex rocks of which the major types are gneiss, pegmatite, pegmatite schist and undifferentiated schist [14]. The soils of the area are Lixosols and Ultisols.

\section{Sampling Stations and Sample Collection}

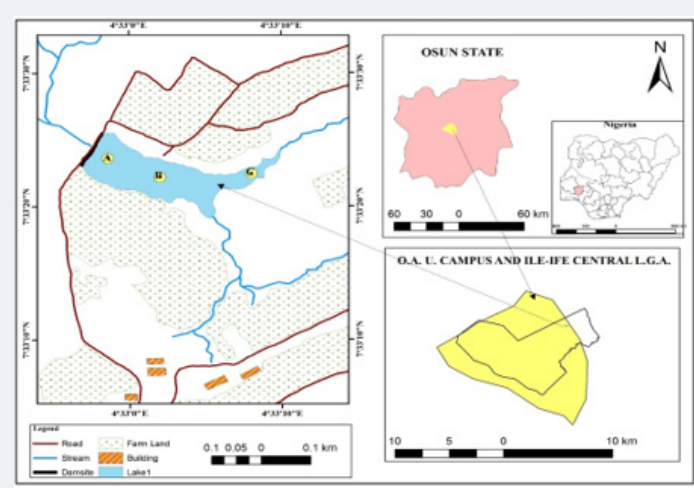

Figure 1: Map of Obafemi Awolowo University (O.A.U.) and lleIfe Central L.G.A. showing Teaching and Research farm Lake 1, O.A.U 
Three sampling stations (Station A, B and C; representing the upper reach, mid basin and dam site respectively) were sited along the horizontal axis of the lake from where samples were collected from surface and at one-meter depth intervals towards the bottom of the lake as indicated (Figure 1). The grid-coordinates of the stations were determined using portable global positioning system (GPS) set and are presented in table 1. The field survey Table 1: Description and grid-coordinates of the Sampling Stations. for this study was conducted monthly for a period of one year (September 2006 to August 2007). An improvised water sampler made of a bottle with heavy stone tied at the bottom to sink the bottle to the desired depth of the lake water and a removable cork as well as a calibrated rope on it was used for sampling subsurface water at the indicated depths of the lake water. (Table 1)

\begin{tabular}{|c|c|c|c|c|c|c|}
\hline $\mathrm{S} / \mathrm{N}$ & Sampling Station & Sampling Depth & Site Description & Latitude (N) & Longitude (E) & Altitude (m) \\
\hline \multirow{4}{*}{1} & AS & Surface & Near the dam & $07^{0} 33.385^{\prime}$ & $004^{0} 32.980^{\prime}$ & 274 \\
\hline & $\mathrm{A} 1$ & $1 \mathrm{~m}$ & & & & \\
\hline & A2 & $2 \mathrm{~m}$ & & & & \\
\hline & $\mathrm{AB}$ & Bottom & & & & \\
\hline \multirow{3}{*}{2} & BS & Surface & Mid lake & $07^{0} 33.373^{\prime}$ & $004^{0} 33.032^{\prime}$ & 275 \\
\hline & B1 & $1 \mathrm{~m}$ & & & & \\
\hline & $\mathrm{BB}$ & Bottom & & & & \\
\hline \multirow{2}{*}{3} & CS & Surface & Near inflow & $07^{0} 33.382^{\prime}$ & $004^{0} 33.129^{\prime}$ & 281 \\
\hline & $\mathrm{CB}$ & Bottom & & & & \\
\hline
\end{tabular}

$\mathrm{m}=$ meters, $\mathrm{N}=$ North, $\mathrm{E}=$ East

Water temperature was measured in-situ using mercury-inglass bulb thermometer with calibration range $\left(-10{ }^{\circ} \mathrm{C}-110{ }^{\circ} \mathrm{C}\right)$, depth and transparency were also measuring in the field (using a Secchi disc). Samples for analysis of the physico-chemical parameters were collected in a clean 2 litres plastic bottles (except samples for Dissolved Oxygen (DO) and Boichemical Oxygen Demand (BOD)). Glass reagent bottles were used to collect samples for DO determination and the samples were fixed on the field immediately after collection with Winkler's reagent (Manganous sulphate and Alkaline Iodide) while dark reagent bottles were used for BOD determination then kept in a dark cupboard for 5 days for subsequent analysis. Sample pH was measured using a Lovibond (1000 - model) pH comparator, while electrical conductivity was measured with (Jenway 4071 model) conductivity meter at $25{ }^{\circ} \mathrm{C}$. The conductivity meter was standardized using a set of potassium chloride ( $\mathrm{KCl}$ ) standard solutions [15].

\section{Laboratory Analyses of Samples}

The analytical determinations of the physico-chemical parameters of the lake water quality were carried out within the holding time of each parameter, following applicable standard methods $[3,16,17]$. The (DO) dissolved oxygen content and Boichemical Oxygen Demand (BOD) were determined by Iodiometric titration. The total solids (TS) as well as total dissolved solids (TDS) of the samples were determined gravimetrically after the samples were oven dried to constant weight at $105 \pm 2$ ${ }^{0} \mathrm{C}$. Total suspended solids (TSS), was calculated as the difference between TS and TDS.

Color was determined calorimetrically using a colorimeter (Jenway 6051 model) standardized with a set of potassium chloroplatinate-cobalt solutions as Pt-Co standards, while turbidity was determined using a turbidimeter with values expressed in nephelometric turbidity units (NTU) [3]. Total acidity, total alkalinity and chloride ions $\left(\mathrm{Cl}^{-}\right)$contents were determined by titrimetric methods while sulphate $\left(\mathrm{SO}_{4}{ }^{2-}\right)$, nitrate $\left(\mathrm{NO}_{3}{ }^{-}\right)$and phosphate $\left(\mathrm{PO}_{4}^{3-}\right)$ ions were determined by spectrophotometric methods. Calcium $\left(\mathrm{Ca}^{2+}\right)$ and magnesium $\left(\mathrm{Mg}^{2+}\right)$ ions were determined by complexio-metric titration method using $\mathrm{Na}_{2}$ EDTA while Sodium $\left(\mathrm{Na}^{+}\right)$and Potassium $\left(\mathrm{K}^{+}\right)$ions were determined by the atomic emission spectrophotometric method using a flame analyzer. All the recommended quality control (QC) and quality assurance $(\mathrm{QA})$ measures were taken for each determination.

The data obtained were subjected to appropriate statistical analyses including descriptive statistics, analysis of variance (ANOVA), cluster analysis and Principal Component Analysis (PCA) using relevant statistical software viz: Paleontological Statistics (PAST), Microsoft Excel, Statistical Product and Service Solutions (SPSS).

\section{Results}

\section{Spatial variations of physico-chemical characteristic of the lake}

There was no significant $(\mathrm{p}>0.05)$ horizontal variation (i.e upstream - dowstream) in the physico-chemical characteristics of the lake as obvious in (Table 2). Vertical variations of the physicochemical parameters revealed that the values of Apparent-colour, True colour, Turbidity, Total Suspended Solids (TSS), Total Solids (TS) and Total Acidity increased significantly $(\mathrm{p}<0.05)$ from (the lake surface $=420.3 \pm 59.4$ Pt-Co; $122.3 \pm 11.8$ Pt-Co; 31.1 $\pm 5.9 \mathrm{NTU} ; 135.0 \pm 22.7 \mathrm{mgL}^{-1} ; 194.3 \pm 23.1 \mathrm{mgL}^{-1} ; 10.87 \pm 0.75$ $\mathrm{mgCaCO}_{3} \mathrm{~L}^{-1}$ ) towards (the bottom $=749.6 \pm 85.2$ Pt-Co; 210.2 \pm 25.2 Pt-Co; $62.9 \pm 7.7$ NTU; $205.4 \pm 22.6 \mathrm{mgL}^{-1} ; 261.2 \pm 22.6$ 
$\left.\mathrm{mgL}^{-1} ; 16.69 \pm 1.17 \mathrm{mgCaCO}_{3} \mathrm{~L}^{-1}\right) .$. In the other hand, water $\mathrm{pH}, \quad \pm 0.05 ; 5.61 \pm 0.24 \mathrm{mgL}^{-1} ; 73.74 \pm 3.13 \mathrm{mgL}^{-1} ; 5.71 \pm 0.55 \mathrm{mgL}^{-1}$ ) Dissolved Oxygen (DO); Dissolved Oxygen Saturation (D0\% Sat.), towards the bottom (6.27 $\pm 0.04 \mathrm{mgL}^{-1} ; 2.35 \pm 0.23 \mathrm{mgL}^{-1} ; 29.69$ Biochemical Oxygen Demand $\left(\mathrm{BOD}_{5}\right)$ in the respective order $\pm 2.91 \mathrm{mgL}^{-1} ; 2.98 \pm 0.61 \mathrm{mgL}^{-1}$ ) of the lake respectively (Table 3). decreased significantly $(\mathrm{p}<0.05)$ from the lake surface $(6.64$

Table 2: Horizontal variation of the physico-chemical characteristics of the lake water

\begin{tabular}{|c|c|c|c|c|c|c|c|}
\hline \multirow[b]{2}{*}{$\mathbf{S} / \mathbf{N}$} & \multirow[b]{2}{*}{ Parameter } & \multirow[b]{2}{*}{ Unit } & \multicolumn{3}{|c|}{ Station } & \multicolumn{2}{|c|}{ Anova } \\
\hline & & & $\begin{array}{c}\text { Upper reach }(n=37) \\
\text { Mean } \pm \text { S.E }\end{array}$ & $\begin{array}{c}\text { Mid basin }(n=26) \\
\text { Mean } \pm \text { S.E }\end{array}$ & $\begin{array}{c}\text { Dam site }(n=13) \\
\text { Mean } \pm \text { S.E }\end{array}$ & $\mathbf{F}$ & $\mathbf{P}$ \\
\hline 1 & Water-Temperature & ${ }^{0} \mathrm{C}$ & $28.0 \pm 0.3$ & $28.4 \pm 0.4$ & $29.2 \pm 0.5$ & 1.763 & 0.179 \\
\hline 2 & Apparent-Colour & Pt-Co & $619.9 \pm 72.0$ & $494.6 \pm 59.5$ & $341.7 \pm 31.9$ & 3.088 & 0.052 \\
\hline 3 & True-Colour & Pt-Co & $168.3 \pm 19.6$ & $158.6 \pm 17.3$ & $161.9 \pm 18.6$ & 0.071 & 0.931 \\
\hline 4 & Turbidity & NTU & $47.7 \pm 6.9$ & $37.3 \pm 5.6$ & $27.1 \pm 3.9$ & 1.913 & 0.155 \\
\hline 5 & $\begin{array}{l}\text { Total Suspended Solids } \\
\text { (TSS) }\end{array}$ & $\mathrm{mgL}^{-1}$ & $164.0 \pm 20.9$ & $152 \pm 22.1$ & $111.0 \pm 11.2$ & 1.079 & 0.345 \\
\hline 6 & Total Solids (TS) & $\mathrm{mgL}^{-1}$ & $220.0 \pm 21.0$ & $208 \pm 22.5$ & $170.0 \pm 13.2$ & 0.896 & 0.413 \\
\hline 7 & $\mathrm{pH}$ & $0-14$ & $6.44 \pm 0.04$ & $6.48 \pm 0.06$ & $6.50 \pm 0.05$ & 0.379 & 0.686 \\
\hline 8 & Conductivity & $\mu \mathrm{Scm}^{-1}$ & $84.00 \pm 3.50$ & $83.00 \pm 3.90$ & $75.00 \pm 2.40$ & 1.185 & 0.312 \\
\hline 9 & $\begin{array}{l}\text { Total Dissolved Solids } \\
\text { (TDS) }\end{array}$ & $\mathrm{mgL}^{-1}$ & $56.00 \pm 1.00$ & $55.00 \pm 2.70$ & $50.00 \pm 2.00$ & 0.868 & 0.424 \\
\hline 10 & Total-Alkalinity & $\mathrm{mgCaCO}_{3} \mathrm{~L}^{-1}$ & $22.10 \pm 0.80$ & $21.90 \pm 0.60$ & $22.20 \pm 0.90$ & 0.029 & 0.971 \\
\hline 11 & Total-Hardness & $\mathrm{mgCaCO}_{3} \mathrm{~L}^{-1}$ & $11.54 \pm 0.77$ & $12.01 \pm 0.98$ & $10.38 \pm 0.74$ & 0.559 & 0.574 \\
\hline 12 & Total-Acidity & $\mathrm{mgCaCO}_{3} \mathrm{~L}^{-1}$ & $14.00 \pm 1.00$ & $13.00 \pm 1.00$ & $12.00 \pm 1.50$ & 0.84 & 0.436 \\
\hline 13 & Calcium ion $\left(\mathrm{Ca}^{2+}\right)$ & $\mathrm{mgL}^{-1}$ & $3.92 \pm 0.26$ & $4.13 \pm 0.31$ & $3.59 \pm 0.26$ & 0.571 & 0.567 \\
\hline 15 & Magnesium ion $\left(\mathrm{Mg}^{2+}\right)$ & $\mathrm{mgL}^{-1}$ & $0.43 \pm 0.06$ & $0.39 \pm 0.08$ & $0.34 \pm 0.08$ & 0.286 & 0.752 \\
\hline 16 & Sodium ion $\left(\mathrm{Na}^{+}\right)$ & $\mathrm{mgL}^{-1}$ & $6.42 \pm 0.16$ & $6.16 \pm 0.17$ & $5.93 \pm 0.18$ & 1.624 & 0.204 \\
\hline 17 & Potassium ion $\left(\mathrm{K}^{+}\right)$ & $\mathrm{mgL}^{-1}$ & $2.56 \pm 0.17$ & $2.49 \pm 0.21$ & $2.11 \pm 0.12$ & 1.022 & 0.365 \\
\hline 18 & $\begin{array}{l}\text { Bicarbonate ion } \\
\left(\mathrm{HCO}_{3}^{-1}\right)\end{array}$ & $\mathrm{mgL}^{-1}$ & $26.50 \pm 1.00$ & $26.20 \pm 0.80$ & $26.60 \pm 1.10$ & 0.029 & 0.971 \\
\hline 19 & Chloride ion $\left(\mathrm{Cl}^{-1}\right)$ & $\mathrm{mgL}^{-1}$ & $4.29 \pm 0.18$ & $4.39 \pm 0.23$ & $4.18 \pm 0.30$ & 0.15 & 0.861 \\
\hline 20 & Sulphate ion $\left(\mathrm{SO}_{4}{ }^{2-}\right)$ & $\mathrm{mgL}^{-1}$ & $4.63 \pm 0.18$ & $4.81 \pm 0.32$ & $4.66 \pm 0.52$ & 0.121 & 0.887 \\
\hline 21 & Dissolved Oxygen (DO) & $\mathrm{mgL}^{-1}$ & $3.60 \pm 0.30$ & $4.20 \pm 0.40$ & $4.60 \pm 0.50$ & 1.803 & 0.172 \\
\hline 22 & $\begin{array}{l}\text { Dissolved Oxygen } \\
\text { Saturation }\end{array}$ & $\%$ & $45.60 \pm 4.00$ & $54.10 \pm 4.90$ & $60.20 \pm 6.60$ & 2.022 & 0.14 \\
\hline 23 & $\begin{array}{l}\text { Biochemical Oxygen } \\
\text { Demand }\left(\mathrm{BOD}_{5}\right)\end{array}$ & $\mathrm{mgL}^{-1}$ & $4.30 \pm 0.60$ & $4.10 \pm 0.60$ & $3.20 \pm 0.30$ & 0.667 & 0.517 \\
\hline 24 & Organic-Matter (OM) & $\mathrm{mgL}^{-1}$ & $8.16 \pm 0.58$ & $7.45 \pm 0.61$ & $7.11 \pm 1.06$ & 0.588 & 0.558 \\
\hline 25 & Nitrate ion $\left(\mathrm{NO}_{3}^{-}\right)$ & $\mathrm{mgL}^{-1}$ & $1.29 \pm 0.14$ & $1.13 \pm 0.12$ & $0.92 \pm 0.09$ & 1.505 & 0.229 \\
\hline 26 & Phosphate $\left(\mathrm{PO}_{4}{ }^{3-}\right)$ & $\mathrm{mgL}^{-1}$ & $0.22 \pm 0.01$ & $0.23 \pm 0.02$ & $0.20 \pm 0.02$ & 0.809 & 0.449 \\
\hline
\end{tabular}

\section{S.E. $=$ Standard Error of Mean}

Table 3: Vertical variation of the physico-chemical characteristics of the lake water

\begin{tabular}{|c|c|c|c|c|c|c|c|}
\hline \multirow{2}{*}{ S/N } & Parameter & \multirow{2}{*}{ Unit } & \multicolumn{3}{|c|}{ Water Level } & \multicolumn{2}{|c|}{ Anova } \\
\cline { 4 - 8 } & & & $\begin{array}{c}\text { Surface }(\mathbf{n}=\mathbf{3 0}) \\
\text { Mean } \pm \text { S.E }\end{array}$ & $\begin{array}{c}\text { Mid-depth(n= 20) } \\
\text { Mean } \pm \text { S.E }\end{array}$ & $\begin{array}{c}\text { Bottom(n= 26) } \\
\text { Mean } \pm \text { S.E }\end{array}$ & F & P \\
\hline 1 & Water-Temperature & ${ }^{0} \mathrm{C}$ & $29.7 \pm 0.3$ & $27.5 \pm 0.3$ & $27.5 \pm 0.3$ & 18.64 & $2.88 \times 10^{-7 * * *}$ \\
\hline 2 & Apparent-Colour & Pt-Co & $420.3 \pm 59.4$ & $406.9 \pm 34.4$ & $749.6 \pm 85.2$ & 8.494 & $0.0005^{* * *}$ \\
\hline 3 & True-Colour & Pt-Co & $122.3 \pm 11.8$ & $166.0 \pm 17.8$ & $210.2 \pm 25.2$ & 6.011 & $0.004^{* *}$ \\
\hline 4 & Turbidity & NTU & $31.1 \pm 5.9$ & $25.9 \pm 2.8$ & $62.9 \pm 7.7$ & 10.23 & $0.0001^{* * *}$ \\
\hline 5 & $\begin{array}{c}\text { Total Suspended Solids } \\
\text { (TSS) }\end{array}$ & $\mathrm{mgL}^{-1}$ & $135.0 \pm 22.7$ & $103.5 \pm 9.7$ & $205.4 \pm 22.6$ & 5.81 & $0.005^{* *}$ \\
\hline
\end{tabular}


Oceanography \& Fisheries Open access Journal

\begin{tabular}{|c|c|c|c|c|c|c|c|}
\hline 6 & Total Solids (TS) & $\mathrm{mgL}^{-1}$ & $194.3 \pm 23.1$ & $156.5 \pm 10.6$ & $261.2 \pm 22.6$ & 5.759 & $0.005^{* *}$ \\
\hline 7 & $\mathrm{pH}$ & $0-14$ & $6.64 \pm 0.05$ & $6.44 \pm 0.04$ & $6.27 \pm 0.04$ & 18.26 & $3.71 \times 10^{-7 * * *}$ \\
\hline 8 & Conductivity & $\mu \mathrm{Scm}^{-1}$ & $83.90 \pm 3.73$ & $79.30 \pm 3.60$ & $82.27 \pm 4.02$ & 0.338 & 0.715 \\
\hline 9 & $\begin{array}{l}\text { Total Dissolved Solids } \\
\text { (TDS) }\end{array}$ & $\mathrm{mgL}^{-1}$ & $55.33 \pm 2.17$ & $52.50 \pm 2.39$ & $55.00 \pm 2.88$ & 0.336 & 0.716 \\
\hline 10 & Total-Alkalinity & $\mathrm{mgCaCO}_{3} \mathrm{~L}^{-1}$ & $21.50 \pm 0.88$ & $23.00 \pm 0.51$ & $21.90 \pm 0.87$ & 0.84 & 0.436 \\
\hline 11 & Total-Hardness & $\mathrm{mgCaCO}_{3} \mathrm{~L}^{-1}$ & $11.29 \pm 0.90$ & $11.49 \pm 0.77$ & $11.76 \pm 0.96$ & 0.074 & 0.928 \\
\hline 12 & Total-Acidity & $\mathrm{mgCaCO}_{3} \mathrm{~L}^{-1}$ & $10.87 \pm 0.75$ & $12.20 \pm 1.17$ & $16.69 \pm 1.17$ & 9.612 & $0.0002^{* * *}$ \\
\hline 13 & Calcium ion $\left(\mathrm{Ca}^{2+}\right)$ & $\mathrm{mgL}^{-1}$ & $3.85 \pm 0.28$ & $3.95 \pm 0.27$ & $4.02 \pm 0.32$ & 0.088 & 0.916 \\
\hline 15 & Magnesium ion $\left(\mathrm{Mg}^{2+}\right)$ & $\mathrm{mgL}^{-1}$ & $0.40 \pm 0.06$ & $0.39 \pm 0.07$ & $0.40 \pm 0.08$ & 0.008 & 0.992 \\
\hline 16 & Sodium ion $\left(\mathrm{Na}^{+}\right)$ & $\mathrm{mgL}^{-1}$ & $6.44 \pm 0.19$ & $6.06 \pm 0.17$ & $6.16 \pm 0.16$ & 1.269 & 0.287 \\
\hline 17 & Potassium ion $\left(\mathrm{K}^{+}\right)$ & $\mathrm{mgL}^{-1}$ & $2.50 \pm 0.19$ & $2.42 \pm 0.21$ & $2.45 \pm 0.20$ & 0.036 & 0.964 \\
\hline 18 & Bicarbonate ion $\left(\mathrm{HCO}_{3}^{-1}\right)$ & $\mathrm{mgL}^{-1}$ & $25.80 \pm 1.10$ & $27.60 \pm 0.60$ & $26.20 \pm 1.00$ & 0.84 & 0.436 \\
\hline 19 & Chloride ion $\left(\mathrm{Cl}^{-1}\right)$ & $\mathrm{mgL}^{-1}$ & $4.20 \pm 0.23$ & $4.22 \pm 0.20$ & $4.49 \pm 0.22$ & 0.552 & 0.578 \\
\hline 20 & Sulphate ion $\left(\mathrm{SO}_{4}{ }^{2-}\right)$ & $\mathrm{mgL}^{-1}$ & $4.49 \pm 0.30$ & $4.98 \pm 0.31$ & $4.71 \pm 0.22$ & 0.713 & 0.493 \\
\hline 21 & Dissolved Oxygen (D0) & $\mathrm{mgL}^{-1}$ & $5.61 \pm 0.24$ & $3.52 \pm 0.25$ & $2.35 \pm 0.23$ & 50.65 & $1.6 \times 10^{-14 * * *}$ \\
\hline 22 & $\begin{array}{l}\text { Dissolved Oxygen Satu- } \\
\text { ration }\end{array}$ & $\%$ & $73.74 \pm 3.13$ & $44.55 \pm 3.10$ & $29.69 \pm 2.91$ & 58.19 & $7.7 \times 10^{-16 * * *}$ \\
\hline 23 & $\begin{array}{l}\text { Biochemical Oxygen } \\
\text { Demand }\left(\mathrm{BOD}_{5}\right)\end{array}$ & $\mathrm{mgL}^{-1}$ & $5.71 \pm 0.55$ & $2.81 \pm 0.23$ & $2.98 \pm 0.61$ & 9.869 & $0.0002^{* * *}$ \\
\hline 24 & Organic-Matter (OM) & $\mathrm{mgL}^{-1}$ & $7.38 \pm 0.64$ & $6.90 \pm 0.74$ & $8.77 \pm 0.65$ & 2.029 & 0.139 \\
\hline 25 & Nitrate ion $\left(\mathrm{NO}_{3}{ }^{-}\right)$ & $\mathrm{mgL}^{-1}$ & $1.16 \pm 0.14$ & $0.91 \pm 0.05$ & $1.39 \pm 0.16$ & 2.813 & 0.067 \\
\hline 26 & Phosphate $\left(\mathrm{PO}_{4}^{3-}\right)$ & $\mathrm{mgL}^{-1}$ & $0.23 \pm 0.01$ & $0.20 \pm 0.02$ & $0.23 \pm 0.02$ & 0.433 & 0.651 \\
\hline
\end{tabular}

S.E. = Standard Error of Mean; ${ }^{* *}=$ highly significant; ${ }^{* * *}=$ very highly significant

\section{Seasonal Variations of Physico-Chemical Characteristic of the Lake}

Table 4: Seasonal variation of the physico-chemical characteristics of the lake water

\begin{tabular}{|c|c|c|c|c|c|c|}
\hline \multirow[b]{2}{*}{ S/N } & \multirow[b]{2}{*}{ Parameter } & \multirow[b]{2}{*}{ Unit } & \multicolumn{2}{|c|}{ Season } & \multicolumn{2}{|c|}{ Anova } \\
\hline & & & $\begin{array}{c}\text { Dry season }(\mathrm{n}=18) \\
\text { Mean } \pm \text { S.E. }\end{array}$ & $\begin{array}{c}\text { Rainy season }(n=58) \\
\text { Mean } \pm \text { S.E. }\end{array}$ & $\mathbf{F}$ & $\mathbf{P}$ \\
\hline 1 & Water-Temperature & ${ }^{\circ} \mathrm{C}$ & $27.9 \pm 0.5$ & $28.5 \pm 0.3$ & 1.345 & 0.25 \\
\hline 2 & Apparent-Colour & Pt-Co & $586.8 \pm 126.3$ & $511.6 \pm 39.7$ & 2.295 & 0.134 \\
\hline 3 & True-Colour & Pt-Co & $105.1 \pm 10.2$ & $182.1 \pm 14.0$ & 8.899 & $0.004^{* *}$ \\
\hline 4 & Turbidity & NTU & $48.0 \pm 12.8$ & $38.3 \pm 3.5$ & 1.059 & 0.307 \\
\hline 5 & Total Suspended Solids (TSS) & $\mathrm{mgL}^{-1}$ & $181.0 \pm 42.8$ & $142.0 \pm 110.5$ & 1.674 & 0.12 \\
\hline 6 & Total Solids (TS) & $\mathrm{mgL}^{-1}$ & $231.0 \pm 42.8$ & $200.0 \pm 10.9$ & 0.993 & 0.322 \\
\hline 7 & $\mathrm{pH}$ & $0-14$ & $6.68 \pm 0.08$ & $6.75 \pm 0.03$ & 13.5 & $0.0004^{* * *}$ \\
\hline 8 & Conductivity & $\mu \mathrm{Scm}^{-1}$ & $73.00 \pm 1.60$ & $85.00 \pm 2.70$ & 6.171 & $0.015^{*}$ \\
\hline 9 & Total Dissolved Solids (TDS) & $\mathrm{mgL}^{-1}$ & $50.00 \pm 1.30$ & $56.00 \pm 1.80$ & 3.089 & 0.083 \\
\hline 10 & Total-Alkalinity & $\mathrm{mgCaCO}_{3} \mathrm{~L}^{-1}$ & $22.10 \pm 1.70$ & $22.00 \pm 0.40$ & 0.017 & 0.898 \\
\hline 11 & Total-Hardness & $\mathrm{mgCaCO}_{3} \mathrm{~L}^{-1}$ & $6.65 \pm 0.49$ & $13.01 \pm 0.52$ & 41.98 & $9.05 \times 10^{-9 * * *}$ \\
\hline 12 & Total-Acidity & $\mathrm{mgCaCO}_{3} \mathrm{~L}^{-1}$ & $12.00 \pm 1.00$ & $14.00 \pm 0.80$ & 0.889 & 0.349 \\
\hline 13 & Calcium ion $\left(\mathrm{Ca}^{2+}\right)$ & $\mathrm{mgL}^{-1}$ & $2.36 \pm 0.19$ & $4.43 \pm 0.17$ & 40.65 & $1.4 \times 10^{-8 * * *}$ \\
\hline 15 & Magnesium ion $\left(\mathrm{Mg}^{2+}\right)$ & $\mathrm{mgL}^{-1}$ & $0.16 \pm 0.02$ & $0.47 \pm 0.05$ & 13.78 & $0.0004^{* * *}$ \\
\hline 16 & Sodium ion $\left(\mathrm{Na}^{+}\right)$ & $\mathrm{mgL}^{-1}$ & $6.29 \pm 0.26$ & $6.22 \pm 0.11$ & 0.114 & 0.737 \\
\hline 17 & Potassium ion $\left(\mathrm{K}^{+}\right)$ & $\mathrm{mgL}^{-1}$ & $1.67 \pm 0.04$ & $2.70 \pm 0.13$ & 18.82 & $4.48 \times 10^{-5 * * *}$ \\
\hline 18 & Bicarbonate ion $\left(\mathrm{HCO}_{3}^{-1}\right)$ & $\mathrm{mgL}^{-1}$ & $26.50 \pm 2.00$ & $26.40 \pm 0.40$ & 0.017 & 0.898 \\
\hline
\end{tabular}




\section{Oceanography \& Fisheries Open access Journal}

\begin{tabular}{|c|c|c|c|c|c|c|}
\hline 19 & Chloride ion $\left(\mathrm{Cl}^{-1}\right)$ & $\mathrm{mgL}^{-1}$ & $4.16 \pm 0.26$ & $4.35 \pm 0.15$ & 0.393 & 0.533 \\
\hline 20 & Sulphate ion $\left(\mathrm{SO}_{4}{ }^{2-}\right)$ & $\mathrm{mgL}^{-1}$ & $3.98 \pm 0.25$ & $4.92 \pm 0.19$ & 6.573 & $0.012^{*}$ \\
\hline 21 & Dissolved Oxygen (DO) & $\mathrm{mgL}^{-1}$ & $5.10 \pm 0.40$ & $3.60 \pm 0.20$ & 10.22 & $0.002^{* *}$ \\
\hline 22 & Dissolved Oxygen Saturation & $\%$ & $65.50 \pm 5.00$ & $46.50 \pm 3.20$ & 8.941 & $0.004^{* *}$ \\
\hline 23 & $\begin{array}{c}\text { Biochemical Oxygen Demand } \\
\left(\mathrm{BOD}_{5}\right)\end{array}$ & $\mathrm{mgL}^{-1}$ & $3.90 \pm 0.90$ & $4.00 \pm 0.40$ & 0.018 & 0.895 \\
\hline 24 & Organic-Matter (OM) & $\mathrm{mgL}^{-1}$ & $7.30 \pm 1.00$ & $7.87 \pm 0.42$ & 0.365 & 0.548 \\
\hline 25 & Nitrate ion $\left(\mathrm{NO}_{3}{ }^{-}\right)$ & $\mathrm{mgL}^{-1}$ & $1.47 \pm 0.29$ & $1.08 \pm 0.05$ & 4.391 & $0.040^{*}$ \\
\hline 26 & Phosphate $\left(\mathrm{PO}_{4}{ }^{3-}\right)$ & $\mathrm{mgL}^{-1}$ & $0.22 \pm 0.02$ & $0.22 \pm 0.01$ & 0.001 & 0.976 \\
\hline
\end{tabular}

S.E. $=$ Standard Error of Mean; ${ }^{*}=$ significant; ${ }^{* *}=$ highly significant; ${ }^{* * *}=$ very highly significant

Dissolved Oxygen (DO); Dissolved Oxygen Saturation (DO\% Sat.) and Nitrate ion $\left(\mathrm{NO}_{3}^{-}\right)$were significantly higher $(\mathrm{p}<0.05)$ in the dry season $\left(5.10 \pm 0.40 \mathrm{mgL}^{-1} ; 65.50 \pm 5.00 \mathrm{mgL}^{-1} ; 1.47 \pm\right.$ $\left.0.29 \mathrm{mgL}^{-1}\right)$ than in the rainy season $\left(3.60 \pm 0.20 \mathrm{mgL}^{-1} ; 46.50 \pm\right.$ $3.20 \mathrm{mgL}^{-1} ; 1.08 \pm 0.05 \mathrm{mgL}^{-1}$ ) respectively. Likewise, True colour, $\mathrm{pH}$, Conductivity, Total Hardness, Calcium ion $\left(\mathrm{Ca}^{2+}\right)$, Magnesium ion $\left(\mathrm{Mg}^{2+}\right)$, Potassium ion $\left(\mathrm{K}^{+}\right)$and Sulphate ion $\left(\mathrm{SO}_{4}{ }^{2-}\right)$ were significantly higher $(\mathrm{p}<0.05)$ in the rainy season: $(182.1 \pm 14.0$ Pt-Co; $6.75 \pm 0.03 ; 85.00 \pm 2.70 \mu \mathrm{Scm}^{-1} ; 13.01 \pm 0.52 \mathrm{mgCaCO}_{3} \mathrm{~L}^{-1}$; $4.43 \pm 0.17 \mathrm{mgL}^{-1} ; 0.47 \pm 0.05 \mathrm{mgL}^{-1} ; 2.70 \pm 0.13 \mathrm{mgL}^{-1} ; 4.92 \pm$ $\left.0.19 \mathrm{mgL}^{-1}\right)$ than in the dry season: $(105.1 \pm 10.2 \mathrm{Pt}-\mathrm{Co} ; 6.68 \pm$ $0.08 ; 73.00 \pm 1.60 \mu \mathrm{Scm}^{-1} ; 6.65 \pm 0.49 \mathrm{mgCaCO}_{3} \mathrm{~L}^{-1} ; 2.36 \pm 0.19$ $\left.\mathrm{mgL}^{-1} ; 0.16 \pm 0.02 \mathrm{mgL}^{-1} ; 1.67 \pm 0.04 \mathrm{mgL}^{-1} ; 3.98 \pm 0.25 \mathrm{mgL}^{-1}\right)$ respectively (Table 4).

\section{Relationships among the sampling stations and the physico-chemical characteristics of the lake}

Table 5: Correlation coefficient matrix showing relationships among the sampling stations based on the physico-chemical parameters of water quality

\begin{tabular}{|c|c|c|c|c|c|c|c|c|c|}
\hline Station & AS & A1 & A2 & AB & BS & B1 & BB & CS & CB \\
\hline AS & X & & & & & & & \\
\hline A1 & 0.981 & $\mathrm{X}$ & & & & & & \\
\hline A2 & 0.981 & 0.988 & $\mathrm{X}$ & & & & & \\
\hline AB & 0.983 & 0.967 & 0.992 & $\mathrm{X}$ & & & & \\
\hline BS & 0.998 & 0.986 & 0.978 & 0.974 & $\mathrm{X}$ & & & \\
\hline B1 & 0.981 & 0.999 & 0.988 & 0.967 & 0.987 & $\mathrm{X}$ & & \\
\hline BB & 0.991 & 0.985 & 0.994 & 0.993 & 0.987 & 0.985 & $\mathrm{X}$ & \\
\hline BB & 0.969 & 0.99 & 0.961 & 0.933 & 0.981 & 0.991 & 0.962 & $\mathrm{X}$ & \\
\hline CB & 0.981 & 0.995 & 0.986 & 0.969 & 0.985 & 0.996 & 0.99 & 0.985 & \\
\hline
\end{tabular}

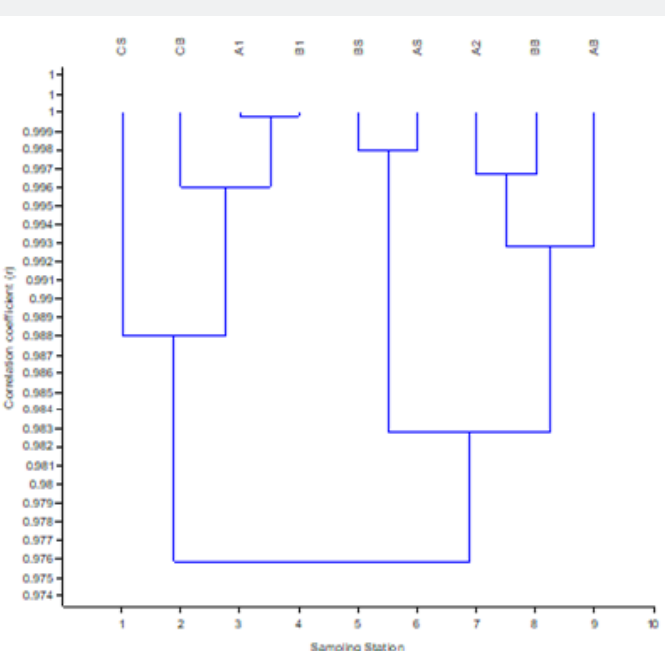

Figure 2: Cluster analysis of the relationships among the Sampling Stations

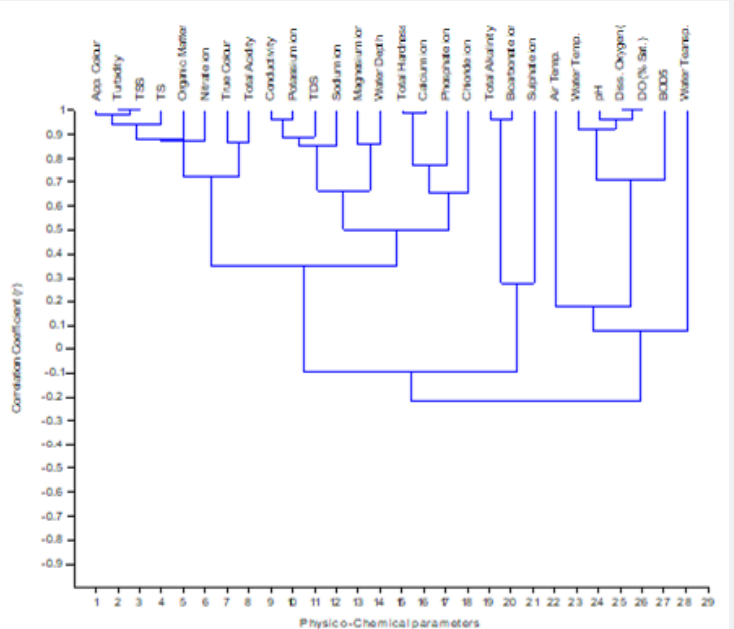

Figure 3: Cluster analysis of the relationships among the physico-chemical characteristics of the lake water 
A relationship among the Sampling Stations based on the physico-chemical characteristics of the lake water is presented in Table 5 (Figure 2). Stations A1 and B1 were the most related forming the first cluster followed by Stations BS and AS; A2 and $\mathrm{BB}$ respectively. Altogether two major clusters were formed (CS, CB, A1, B1) and (BS, AS, A2, BB, AB). All the Sampling Stations showed significant $(p<0.05)$ positive relationships based on the physico-chemical contents of the water samples (Figure 3).

Figure 3 is a cluster analysis of the relationships among the physico-chemical characteristics of the lake. Altogether five major clusters were formed; the clusters (Apparent Colour, Turbidity, TSS, TS, Organic Matter, Nitrate ion, True Colour, Total Acidity) and (Conductivity, Potassium ion, TDS, Sodium ion, Magnesium ion, Water Depth) as well as (Total Hardness, Calcium ion, Phosphate ion, Chloride ion) were significantly ( $\mathrm{p}<0.05$ ) positively correlated. The cluster (Total Alkalinity, Bicarbonate ion, Sulphate ion) showed positive non-significant $(p>0.05)$ relationship with the first three clusters while (Water Temperature, pH, DO, DO \% Saturation, $\mathrm{BOD}_{5}$, Water Transparency) showed inverse but nonsignificant $(p>0.05)$ relationships with the other four clusters.

\section{Comparison of the values of the physico-chemical characteristics of the lake water with some Standard Water Quality Guides}

Table 6: Comparison of the values of the physico-chemical characteristics of the lake water with some Standard Water Quality Guides (NIS, 2007 and Meade, 1989).

\begin{tabular}{|c|c|c|c|c|c|c|}
\hline$S / N$ & Parameter & Unit & Range & $\begin{array}{l}\text { Current Study } \\
\text { Mean } \pm \text { S.E }\end{array}$ & $\begin{array}{l}\text { Drinking Water Quality } \\
\text { (NIS, 2007) (MPL) }\end{array}$ & $\begin{array}{l}\text { Water Quality for Fish/ } \\
\text { Aquatic life } \\
\text { (Meade, 1989) }\end{array}$ \\
\hline 1 & Water-Temperature & ${ }^{0} \mathrm{C}$ & $24.0-33.5$ & $28.3 \pm 0.2$ & Ambient & - \\
\hline 2 & Apparent-Colour & Pt-Co & $181.1-1826.3$ & $529.4 \pm 42.2$ & $15 \mathrm{TCU}$ & - \\
\hline 3 & True-Colour & Pt-Co & $37.6-643.5$ & $165.2 \pm 11.6$ & $15 \mathrm{TCU}$ & - \\
\hline 4 & Turbidity & NTU & $7.6-183.3$ & $40.6 \pm 4.0$ & 5 & - \\
\hline 5 & $\begin{array}{l}\text { Total Suspended Solids } \\
\text { (TSS) }\end{array}$ & $\mathrm{mgL}^{-1}$ & $40-550$ & $151 \pm 12.8$ & ND & $<80$ \\
\hline 6 & Total Solids (TS) & $\mathrm{mgL}^{-1}$ & $90-590$ & $207 \pm 13.1$ & ND & - \\
\hline 7 & ${ }^{*} \mathrm{pH}$ & $0-14$ & $6.00-6.85$ & $6.38 \pm 0.09$ & $6.5-8.5$ & $6.5-8.0$ \\
\hline 8 & Conductivity & $\mu \mathrm{Scm}^{-1}$ & $58-130$ & $82 \pm 2.2$ & 1000 & - \\
\hline 9 & $\begin{array}{l}\text { Total Dissolved Solids } \\
\text { (TDS) }\end{array}$ & $\mathrm{mgL}^{-1}$ & $40-90$ & $54 \pm 1.5$ & 500 & $<400$ \\
\hline 10 & * Total-Alkalinity & $\mathrm{mgCaCO}_{3} \mathrm{~L}^{-1}$ & $8.0-28.0$ & $22.0 \pm 0.5$ & ND & $10-400$ \\
\hline 11 & * Total-Hardness & $\mathrm{mgCaCO}_{3} \mathrm{~L}^{-1}$ & $0.93-23.31$ & $11.51 \pm 0.52$ & 150 & $10-400$ \\
\hline 12 & Total-Acidity & $\mathrm{mgCaCO}_{3} \mathrm{~L}^{-1}$ & $4.0-30.0$ & $13 \pm 0.7$ & ND & - \\
\hline 13 & Calcium ion $\left(\mathrm{Ca}^{2+}\right)$ & $\mathrm{mgL}^{-1}$ & $0.19-7.57$ & $3.94 \pm 0.17$ & ND & $4-160$ \\
\hline 15 & Magnesium ion $\left(\mathrm{Mg}^{2+}\right)$ & $\mathrm{mgL}^{-1}$ & $0.11-1.52$ & $0.40 \pm 0.04$ & 0.2 & $<15$ \\
\hline 16 & Sodium ion $\left(\mathrm{Na}^{+}\right)$ & $\mathrm{mgL}^{-1}$ & $5.09-9.34$ & $6.24 \pm 0.09$ & 200 & 75 \\
\hline 17 & Potassium ion $\left(\mathrm{K}^{+}\right)$ & $\mathrm{mgL}^{-1}$ & $1.42-5.17$ & $2.46 \pm 0.11$ & ND & $<5$ \\
\hline 18 & $\begin{array}{l}\text { Bicarbonate ion } \\
\left(\mathrm{HCO}_{3}^{-1}\right)\end{array}$ & $\mathrm{mgL}^{-1}$ & $9.6-33.6$ & $26.4 \pm 0.6$ & ND & - \\
\hline 19 & Chloride ion $\left(\mathrm{Cl}^{-1}\right)$ & $\mathrm{mgL}^{-1}$ & $1.14-6.94$ & $4.31 \pm 0.13$ & 250 & - \\
\hline 20 & Sulphate ion $\left(\mathrm{SO}_{4}{ }^{2-}\right)$ & $\mathrm{mgL}^{-1}$ & $1.91-9.92$ & $5.14 \pm 0.41$ & 100 & $<50$ \\
\hline 21 & $\begin{array}{l}\text { *Dissolved Oxygen } \\
\text { (DO) }\end{array}$ & $\mathrm{mgL}^{-1}$ & $0.0-8.0$ & $4.0 \pm 0.2$ & ND & 5 \\
\hline 22 & $\begin{array}{l}\text { Dissolved Oxygen } \\
\text { Saturation }\end{array}$ & $\%$ & $0.0-105.5$ & $51.0 \pm 2.8$ & ND & - \\
\hline 23 & $\begin{array}{l}\text { Biochemical Oxygen } \\
\text { Demand }\left(\mathrm{BOD}_{5}\right)\end{array}$ & $\mathrm{mgL}^{-1}$ & $0.4-16.0$ & $4.0 \pm 0.3$ & ND & - \\
\hline 24 & Organic-Matter (OM) & $\mathrm{mgL}^{-1}$ & $2.03-17.23$ & $7.75 \pm 0.40$ & 5 & - \\
\hline 25 & Nitrate ion $\left(\mathrm{NO}_{3}^{-}{ }^{-}\right)$ & $\mathrm{mgL}^{-1}$ & $0.55-4.70$ & $1.17 \pm 0.08$ & 50 & $0-3$ \\
\hline 26 & Phosphate $\left(\mathrm{PO}_{4}{ }^{3}\right)$ & $\mathrm{mgL}^{-1}$ & $0.10-0.56$ & $0.22 \pm 0.01$ & ND & - \\
\hline
\end{tabular}

$\mathrm{ND}=$ No Data; $\mathrm{MPL}=$ Maximum Permitted Levels; S.E. $=$ Standard Error

* $=$ denotes most common water quality characteristics which will influence fish health and growth (Meade, 1989) 
Except for the Apparent Colour, True Colour, Turbidity that were not within the permissible levels of the NIS, Meade $[18,19]$ values for drinking water and fish/aquatic life respectively, the other phisico-chemical parameters of the lake water were very much within the prescribed limits (Table 6).

\section{Comparison between the results the present study with previous works on the lake}

Table 7: Comparison between the results the present study with previous works on the lake

\begin{tabular}{|c|c|c|c|c|c|c|c|}
\hline \multirow{2}{*}{$\mathbf{S} / \mathbf{N}$} & \multirow{2}{*}{ Parameter } & \multirow{2}{*}{ Unit } & \multicolumn{2}{|c|}{ Previous Studies } & \multicolumn{2}{|c|}{ Current Study } & \multirow{2}{*}{ References } \\
\hline & & & Range & Mean \pm S.E. & Range & Mean \pm S.E. & \\
\hline 1 & Depth & $\mathrm{m}$ & $1.37-5.70$ & $3.54 \pm 2.17$ & $0.16-4.07$ & $2.25 \pm 0.18$ & $\begin{array}{l}\text { Aderounmu \& } \\
\text { Adeniyi [5] }\end{array}$ \\
\hline 2 & Transparency & $\mathrm{m}$ & $0.5-0.9$ & $0.65 \pm 0.04$ & $0.16-1.12$ & $0.69 \pm 0.05$ & $\begin{array}{c}\text { Imevbore et al. } \\
{[26]}\end{array}$ \\
\hline 3 & Water-Temperature & ${ }^{0} \mathrm{C}$ & $26.5-30.0$ & $28.8 \pm 0.5$ & $24.0-33.5$ & $28.3 \pm 0.2$ & $\begin{array}{c}\text { Imevbore et al. } \\
{[26]}\end{array}$ \\
\hline 4 & Apparent-Colour & Pt-Co & - & - & $181.1-1826.3$ & $529.4 \pm 42.2$ & - \\
\hline 5 & True-Colour & Pt-Co & - & - & $37.6-643.5$ & $165.2 \pm 11.6$ & - \\
\hline 6 & Turbidity & NTU & - & - & $7.6-183.3$ & $40.6 \pm 4.0$ & - \\
\hline 7 & $\begin{array}{l}\text { Total Suspended Solids } \\
\text { (TSS) }\end{array}$ & $\mathrm{mgL}^{-1}$ & - & - & $40-550$ & $151 \pm 12.8$ & - \\
\hline 8 & Total Solids (TS) & $\mathrm{mgL}^{-1}$ & - & - & $90-590$ & $207 \pm 13.1$ & - \\
\hline 9 & $* \mathrm{pH}$ & $0-14$ & $6.40-7.70$ & $7.02 \pm 0.24$ & $6.00-6.85$ & $6.38 \pm 0.09$ & $\begin{array}{c}\text { Imevbore et al. } \\
{[26]}\end{array}$ \\
\hline 10 & Conductivity & $\mu \mathrm{Scm}^{-1}$ & $93.1-112.4$ & $103.9 \pm 2.8$ & $58-130$ & $82 \pm 2.2$ & $\begin{array}{c}\text { Imevbore et al. } \\
{[26]}\end{array}$ \\
\hline 11 & $\begin{array}{l}\text { Total Dissolved Solids } \\
\text { (TDS) }\end{array}$ & $\mathrm{mgL}^{-1}$ & - & - & $40-90$ & $54 \pm 1.5$ & - \\
\hline 12 & ${ }^{*}$ Total-Alkalinity & $\mathrm{mgCaCO}_{3} \mathrm{~L}^{-1}$ & $0.166-0.272$ & $0.217 \pm 0.009$ & 28-Aug & $22.0 \pm 0.5$ & $\begin{array}{c}\text { Imevbore et al. } \\
{[26]}\end{array}$ \\
\hline 13 & * Total-Hardness & $\mathrm{mgCaCO}_{3} \mathrm{~L}^{-1}$ & - & - & $0.930-23.31$ & $11.51 \pm 0.52$ & - \\
\hline 15 & Total-Acidity & $\mathrm{mgCaCO}_{3} \mathrm{~L}^{-1}$ & - & - & 30-Apr & $13 \pm 0.7$ & - \\
\hline 16 & Calcium ion $\left(\mathrm{Ca}^{2+}\right)$ & $\mathrm{mgL}^{-1}$ & $5.90-6.90$ & $6.58 \pm 0.15$ & $0.19-7.57$ & $3.94 \pm 0.17$ & $\begin{array}{c}\text { Imevbore et al. } \\
{[26]}\end{array}$ \\
\hline 17 & Magnesium ion $\left(\mathrm{Mg}^{2+}\right)$ & $\mathrm{mgL}^{-1}$ & $3.20-4.10$ & $3.58 \pm 0.17$ & $0.11-1.52$ & $0.40 \pm 0.04$ & $\begin{array}{c}\text { Imevbore et al. } \\
{[26]}\end{array}$ \\
\hline 18 & Sodium ion $\left(\mathrm{Na}^{+}\right)$ & $\mathrm{mgL}^{-1}$ & $2.40-3.80$ & $2.97 \pm 0.24$ & $5.09-9.34$ & $6.24 \pm 0.09$ & $\begin{array}{c}\text { Imevbore et al. } \\
{[26]}\end{array}$ \\
\hline 19 & Potassium ion $\left(\mathrm{K}^{+}\right)$ & $\mathrm{mgL}^{-1}$ & $3.20-4.70$ & $3.83 \pm 0.27$ & $1.42-5.17$ & $2.46 \pm 0.11$ & $\begin{array}{c}\text { Imevbore et al. } \\
{[26]}\end{array}$ \\
\hline 20 & $\begin{array}{c}\text { Bicarbonate ion }\left(\mathrm{HCO}_{3}^{-}\right. \\
1)\end{array}$ & $\mathrm{mgL}^{-1}$ & - & - & $9.6-33.6$ & $26.4 \pm 0.6$ & - \\
\hline 21 & Chloride ion $\left(\mathrm{Cl}^{-1}\right)$ & $\mathrm{mgL}^{-1}$ & $4.30-6.70$ & $5.47 \pm 0.38$ & $1.14-6.94$ & $4.31 \pm 0.13$ & $\begin{array}{c}\text { Imevbore et al. } \\
{[26]}\end{array}$ \\
\hline 22 & Sulphate ion $\left(\mathrm{SO}_{4}{ }^{2-}\right)$ & $\mathrm{mgL}^{-1}$ & - & - & $1.91-9.92$ & $5.14 \pm 0.41$ & - \\
\hline 23 & *Dissolved Oxygen (DO) & $\mathrm{mgL}^{-1}$ & - & - & $0.0-8.0$ & $4.0 \pm 0.2$ & - \\
\hline 24 & $\begin{array}{l}\text { Dissolved Oxygen } \\
\text { Saturation }\end{array}$ & $\%$ & - & - & $0.0-105.5$ & $51.0 \pm 2.8$ & - \\
\hline 25 & $\begin{array}{l}\text { Biochemical Oxygen } \\
\text { Demand }\left(\mathrm{BOD}_{5}\right)\end{array}$ & $\mathrm{mgL}^{-1}$ & - & - & $0.4-16.0$ & $4.0 \pm 0.3$ & - \\
\hline 26 & Organic-Matter (OM) & $\mathrm{mgL}^{-1}$ & $1.03-4.24$ & - & $2.03-17.23$ & $7.75 \pm 0.40$ & *Alamina [42] \\
\hline 27 & Nitrate ion $\left(\mathrm{NO}_{3}^{-}\right)$ & $\mathrm{mgL}^{-1}$ & - & - & $0.55-4.70$ & $1.17 \pm 0.08$ & - \\
\hline 28 & Phosphate $\left(\mathrm{PO}_{4}^{3-}\right)$ & $\mathrm{mgL}^{-1}$ & $0.012-0.140$ & $0.075 \pm 0.025$ & $0.10-0.56$ & $0.22 \pm 0.01$ & $\begin{array}{c}\text { Imevbore et al. } \\
{[26]}\end{array}$ \\
\hline
\end{tabular}

S.E: Standard Error of Mean; ${ }^{*}=$ Alamina, 1969 cited by Hall (1969). 
Comparing the current values of some of the physico-chemical characteristics of the lake water to the previous works on the lake; it is obvious that the nutrients parameters (Organic matter and Phosphate) have increased over time. Meanwhile, Depth, $\mathrm{pH}$, Conductivity, Cations and Anions $\left(\mathrm{Ca}^{2+}, \mathrm{Mg}^{2+}, \mathrm{Na}^{+}, \mathrm{K}^{+}\right.$and $\mathrm{HCO}_{3}$ ) have all reduced in their values from the period of 1969 till present 2006 - 2007 (Table 7).

\section{Discussion}

The significant increase $(\mathrm{P}<0.05)$ in the mean values of apparent colour, true colour, turbidity, TSS and TS from the surface of the lake to the bottom of the lake could be due to resuspension of deposits materials from the bottom of the lake during sampling. For instance, resuspension of (organic particles: decomposed plant and animal matter as well as living organisms e.g. plankton) and/ or (inorganic particles: silt, clay and some chemical compounds) along the vertical column of the lake. The significant $(p<0.05)$ decrease in the hydrogen ion concentration $(\mathrm{pH})$ values of water samples from surface water level to the bottom of the lake and the corresponding significant $(p<0.05)$ increase in the acidity values from surface water level to the bottom of the lake have also been reported in some lakes/reservoirs by some authors. For instance, in Eleiyele Reservoir, Opa Reservoir, Erinle Lake and its major inflows, Guma dam in Sierra Leone and Russell Pond, Woodstock, New Hampshire USA [9,20-23].

Such downward decrease in $\mathrm{pH}$ seems to be a common limnological phenomenon in lakes and probably because the top levels of water column usually comprise the photosynthetic zone (trophogenic layer) while the bottom column is tropholytic and characterised by relatively high organic matter decomposition and the release of carbon dioxide. A decrease in $\mathrm{pH}$ often imply decrease in photosynthetic activities and this is usually coupled with increased concentration of carbon dioxide and other bye products of organic matter decomposition at the lake bottom [24]. The increase in acidic condition with depth could also, be attributed to build-up of carbon dioxide from respiring organisms in the water column as respiration often exceeds production of oxygen by primary producers at these depths. The carbon dioxide produced by respiring organisms combines with water molecules to produce carbonic acid leading to increase in acidic condition.

Significant $(\mathrm{p}<0.05)$ gradual decrease in dissolved oxygen (DO) and DO (\% Sat.) concentrations, along the water column from surface towards the bottom of the lake could be linked with higher photosynthetic activities at the surface and/or the higher decomposition processes at the bottom of the lake [25]. There was total oxygen depletion (anoxic condition) at the bottom of the lake in April 2007. Akinbuwa [24] and Mtada [22] respectively recorded similar anoxic conditions from Opa Reservoir and Guma dam at different times of the year of their studies. Also, worthy of note is the level of oxygen saturation in the lake which was mostly below $100 \%$. This trend of under saturation of oxygen was also recorded for Eleiyele Reservoir, River Oshun, The Blue Nile, and River Sokoto [26-29]. Egborge [27] and Holden and Green [29] explained that the conditions of under saturation were associated with high iron concentration as oxygen is involved in the oxidation of ferrous iron to ferric iron.

High amounts of iron are associated with anaerobic conditions [30]. This was probably the situation in the studied lake in view of its high iron contents (from $0.00-20.20 \mathrm{mgl}^{-1}$ ) with overall mean of $3.99 \mathrm{mgl}^{-1}$. Ekpenyong [31] explained that some insoluble forms of iron such as ferric hydroxide could be present in the lake that could not be detected and may reduce the iron content but also contributed to reduction in oxygen saturation. Supersaturation was found to be a constant feature of the Volta River with considerably low iron content. Akinbuwa [21] also, recorded similar condition of supersaturation with no anoxic condition at the bottom of Erinle Lake, and explained was due to low iron content of the lake. The vertical variation in similar manner of the Biochemical oxygen Demand $\left(\mathrm{BOD}_{5}\right)$ could be because life processes and organic activities were more pronounced at the surface mainly within the euphotic zone. Akinbuwa [21] observed similar trend in vertical variation of $\left(\mathrm{BOD}_{5}\right)$ in Erinle Lake.

Significantly, higher $(\mathrm{p}<0.05)$ values of oxygen concentrations (DO and DO\%Sat.) during the dry season than in the rainy season is in line with the observation on six impoundments on River Oshun by Ayodele \& Adeniyi [32] but contrast the results of Yusoff et al. [33] on Kenyir Reservoir, Malaysia. Higher record of oxygen concentration during the dry season than in the rainy season as recorded in this present study could probably be due to higher photosynthetic activities during the dry season when there is normally more solar energy available to the lake than during the rainy season. Likewise, higher values of nitrate $\left(\mathrm{NO}_{3}^{-}\right)$in the dry season could be because of reduction in the water volume during the dry season and the dilution effect of precipitation and flooding during the rainy season.

Significantly, higher $(\mathrm{p}<0.05)$ true colour values in the rainy season could be because of vegetation decay and higher influx into the basin during the rainy season. The lower $\mathrm{pH}$ values (i.e. more acidic) during the rainy season than in the dry season recorded in this study was like the observations on six impoundments on River Oshun, Southern Nigeria [32]. This could be attributed to the influx of acidic flood water, resuspension of bottom sediment during the rains in contrast to evaporation of water, concentration of calcium salts as well as increased photosynthetic consumption of carbon dioxide during the dry season as suggested by Imevbore [20], Talling and Rhoska [28], Holden and Green [29], Akinbuwa [24], and Umaru [34].

Higher conductivity values measured from the lake water during the rainy season could be attributed to run-off from the surrounding agricultural land. Run-off from agricultural land is suspected to be chief contribution to solids and dissolved ions in lakes and rivers, which in turns usually bring about corresponding increase in the conductivity values. This can also be corroborated by the similar significant higher concentrations of some major cations $\left(\mathrm{Ca}^{2+}, \mathrm{Mg}^{2+}\right.$ and $\left.\mathrm{K}^{+}\right)$and anion $\left(\mathrm{SO}_{4}{ }^{2-}\right)$ during the rainy 
season than in the dry season. Conductivity is a measure of the ability of water to conduct electric current. Current is conducted in aqueous solution by the movement of ions (cations and anions) in water which depends on their total concentration, valence and on the water temperature. It is also noteworthy that the conductivity values of the lake fell within the low-end range of known values for African lake waters being less than $600 \mu \mathrm{Scm}^{-1}$ [27].

The lake thus belongs to Class 1 of the African waters about conductivity values. Class 1 waters are generally poor in chemical nutrients. Similarly, the corresponding significant higher values of total hardness during the rainy season is expected as calcium and magnesium have been noted to be the most common sources of water hardness [35]. The positive significant $(p<0.05)$ relationships among the sampling stations as obvious in the cluster analysis of the relationships among the sampling stations is an indication of the fact that the sampling stations were similar in their physico-chemical properties. This is a common phenomenon in most small shallow lakes.

The water from the lake could be regarded as being relatively clean as the physical parameters (apparent colour, true colour and turbidity) were the only parameters having their overall mean values higher than the prescribed standards limits for both drinking water and water for fish and aquatic life. Sources of colour in waterbodies could be from phytoplankton blooms, decayed vegetal materials as well as presence or introduction of fluvic and humic acid from microbial degradation of dead organisms (Nova Scotia Environment, 2008). Turbidity could result from allochthonous and autochthonous weathered suspended materials in the water. It is a measure of the cloudiness and murkiness of water due to suspended particles. The shallower the waterbody, the much easier for re-suspension of suspended materials in the water that could cause an increase in the turbidity of the water [36]. However, the lake water will require minimal treatment for its suitability for drinking because high colour and turbidity values may indicate potential risks for consumption.

There should also be adequate cares and control measures for its suitability for agriculture mostly fish culture as high colour and turbidity values could all adversely affect the organisms living within the waterbody by interfering with their food chain as well as releasing pollutants in the aquatic ecosystem. Increments in the nutrient compounds (Organic matter and Phosphate) and the corresponding decrease in Depth, $\mathrm{pH}$, Conductivity, Cations and Anions $\left(\mathrm{Ca}^{2+}, \mathrm{Mg}^{2+}, \mathrm{Na}^{+}, \mathrm{K}^{+}\right.$and $\left.\mathrm{HCO}_{3}^{-}\right)$over time, i.e. from the period of 1969 till present $2006-2007$ is an indication of the fact that the lake is aging. Similar changes in these recorded parameters and phenomena have been used as an indication of eutrophication in lakes and other water bodies by various authors [37-43].

\section{Conclusion}

The Obafemi Awolowo University Teaching and Research Farm Lake could be regarded as slightly acidic, soft and wellbuffered fresh-waterbodies. The lake is shallow, coloured, turbid and less transparent. There is evidence of gradual deposits of allochthonous materials from the catchment basin transforming the lake from the original oligotrophic status to a eutrophic waterbody.

\section{References}

1. Tebbutt T H Y (1983) Principle of Water Quality Control. In: $3^{\text {rd }}$ (Edn), Pergamon Press Ltd, Oxford, UK, p. 235.

2. Parashar C, Dixit S, Shrivastava R (2006) Seasonal Variations in Physico-chemical Characteristics in Upper Lake of Bhopal. Asian J Exp Sci 20(2): 297-302.

3. Ademoroti CMA (1996b) Standard Methods for Water and Effluents analysis. In: Foludex Press Ltd, Ibadan, Nigeria, p. 182.

4. Adeniyi F (1971) A Preliminary Ecological Study of the Parasite Fauna of Fishes from the Farm Pond, University of Ife, Nigeria. Unpublished B. Sc. Thesis, Department of Zoology, OAU, Ile-Ife, Nigeria, p. 56.

5. Aderounmu E A and Adeniyi F (1972) Cestodes in Fish from a Pond at Ile-Ife, Nigeria. The African Journal of Tropical Hydrobiology and Fisheries 2: 151-156.

6. Adeniyi I F and Olabanji I O (2005) The physico-chemical and bacteriological quality of rainwater collected over different roofing materials in Ile-Ife, Southwestern Nigeria. Chemistry and Ecology. 21(3): 149-166.

7. Papadakis J (1961) Crop Ecology in West Africa. FAO 2(1).

8. Ojo O (1977) The Climates of West Africa. Heinemann Educational Books Ltd.

9. Akinbuwa O and Adeniyi I F (1996) Seasonal variation, distribution and inter-relationships of rotifers in Opa Reservoir, Nigeria. Afr J Ecol 34(4): 351-363.

10. Muoghalu J I and Johnson S O (2000) Interception, $\mathrm{pH}$ and soil content of rainfall in a Nigerian lowland rainforest. J Ecol 38: 38-46.

11. Keay R W J (1959) An outline of Nigerian vegetation. In: $3^{\text {rd }}($ edn), Government Printer, Nigeria.

12. Onochie C F A (1979) The Nigerian rainforest ecosystem. In: Okali A U U (eds.): The Nigerian Rainforest Ecosystem; Proceedings of the man and biosphere on the Nigerian Rainforest Ecosystem. University of Ibadan, Nigeria National MAB Committee, Ibadan, Nigeria. p. 1-13.

13. Olabanji I 0 and Adeniyi I F (2005) Trace metals in bulk freefall and roof intercepted rainwater at Ile-Ife, Southwest Nigeria. Chemistry and Ecology 21(3): 167-179.

14. Ako B D, Adeniyi I F and Adepoju I F (1990) Statistical tests and chemical quality of shallow groundwater from a metamorphic terrain, Ile-Ife/Modakeke, South Western Nigeria. Journal of Africa Earth Sciences 10(4): 603-613.

15. Golterman R I, Clymo R S and Ohnstad M A M (1978) Methods for physical and chemical analysis of freshwater. IBP Handbook No. 8. Blackwell Scientific Publication, Oxford, UK, P. 214.

16. APHA, AWWA and WEF (1995) Standard methods for the examination of water and wastewater. In: $19^{\text {th }}(\mathrm{edn})$ Washington, USA

17. USEPA (1983) Methods for chemical analysis of water and wastes. EMSL, United State Environmental Protection Agency, Cincinnati, US

18. Nigerian Industrial Standards (NIS) (2007) Nigerian Standard for Drinking Water Quality, p. 30.

19. Meade J W (1989) Aquaculture Management. In: Chapman \& Hall, p. 175.

20. Imevbore A M A (1967) Hydrology and plankton of Eleiyele reservoir Ibadan, Nigeria. Hydrobiologia 30(10): 154-176. 
21. Akinbuwa 0 (1999) The rotifera fuana and physico-chemical conditions of Erinle Lake and its major inflows at Ede, Osun State, Nigeria. In: Zoology Department Obafemi Awolowo University, Ile-Ife, Nigeria. p. 333.

22. Mtada O S M (1988): Observations on the limnology of a tropical reservoir in West Africa (The Guma Dam, Sierra Leone). Part II: Factors influencing its limnochemistry. Tropical Ecology 29(2): 50-60.

23. Bailey M and Davignon T (1999) A limnological assessment of Russell Pond, Woodstock, New Hampshire. UNH center for Freshwater Biology Research 1(2): 13-22.

24. Akinbuwa O (1988) The study of the physic-chemical factors and rotifera fauna of Opa Reservoir, Ile-Ife, Nigeria. Zoology Department, University of Ife, Ile-Ife, Nigeria. p. 261.

25. Arumugam P T and Furtado J I (1980) Physico-chemistry, destratification and nutrient budget of a lowland eutrophicated Malaysian reservoir and its limnological implications. Hydrobiologia 70: 11-24.

26. Imevbore A M A, Meszes G and Boszormenyi Z (1972) The primary productivity of a fish-pond at Ile-Ife, Nigeria. In: Kajak Z and HillbrichtIlkowska A (eds.). Productivity Problems of Freshwaters. Proceedings of the IBP-UNESCO symposium, pp. 715-723.

27. Egborge A B M (1971) The chemical hydrology of the River Oshun, Western State, Nigeria. Fresh Water Biol 1(3): 257-271.

28. Talling J F and J Rzoska (1967) The development of plankton in relation to hydrological regime in the Blue Nile. J Ecol 55: 637-662.

29. Holden M J and Green J (1960) The hydrology and plankton of River Sokoto. J Animal Ecol 29: 65-84.

30. Krenkel P A and Novotny V (1980) Water Quality Management. In: Academic Press, New York, US, p. 671.

31. Ekpenyong H (1982) The physico-chemical quality of water in relation to primary production of fish ponds in Ile-Ife, Oyo State, Nigeria. Unpublished M. Sc. Thesis, University of Ife, Nigeria, p. 23.

32. Ayodele H A and Adeniyi I F (2006) The zooplankton fauna of six impoundments on river Osun, South West, Nigeria. The Zoologist 1(4): 49-67.
33. Yusoff F M, Matias H M and Khan N (2002) Changes of water quality, chlorophyll and zooplankton along the river-lacustrine continuum in a tropical reservoir. Verh Internat Verein Limnol 28: 295-298.

34. Umaru D D (1990) The study of physic-chemical parameters in ABU Pond and Federal Government Demonstration Fish Farm at Mando Road, Kaduna. Unpublished B. Sc. Dissertation, Nigerian Defence Academy, Kaduna, Nigeria.

35. Wurts W A and Durborow R M (1992) Interactions of $\mathrm{pH}$, carbon dioxide, alkalinity and hardness in fishponds. Southern Regional Aquaculture Center 464.

36. (MWSMH) Manitoba Water Stewardship and Manitoba Health (2011) Turbidity in Manitoba Water Supplies. Manitoba Water Stewardship's Office of Drinking Water.

37. Popp A, Hoagland K D and Hergenrader G L (1996) Zooplankton community response to reservoir aging. Hydrobiologia. 339(1-3): 1321.

38. Vollenweider R A (1980) Eutrophication control. Nature and Resources XVI (3): 9-14.

39. Matsumura-Tundisi T, Galizia-Tundisi J and Rocha 0 (2002) Zooplankton diversity in eutrophic systems and its relation to the occurrence of cyanophycean blooms. Verh Internat Verein Limnol 28: 671-674.

40. Winner R W (1972) An Evaluation of Certain Indices of Eutrophy and Maturity in Lakes. Hydrobiologia 40(2): 223-245.

41. Cole G A (1975) Textbook of Limnology. In: The C V Mosby Company, US, p. 283

42. Hall JB (1969) Plant Ecology and taxonomy. Ife University herbarium bulletin 1: 36-37.

43. Nova Scotia Environment (2008): The drop on water colour. The Nova Scotia Environment p. 8.

\section{Your next submission with Juniper Publishers will reach you the below assets}

- Quality Editorial service

- Swift Peer Review

- Reprints availability

- E-prints Service

- Manuscript Podcast for convenient understanding

- Global attainment for your research

- Manuscript accessibility in different formats

( Pdf, E-pub, Full Text, Audio)

- Unceasing customer service

Track the below URL for one-step submission

https://juniperpublishers.com/online-submission.php 